(1)

CrossMark

\title{
Advancing global programmatic management of latent tuberculosis infection for at risk populations
}

\author{
Haileyesus Getahun ${ }^{1}$, Alberto Matteelli², Ibrahim Abubakar ${ }^{3}$, Barbara Hauer $^{4}$, \\ Emanuele Pontali ${ }^{5}$ and Giovanni Battista Migliori ${ }^{6}$
}

Affiliations: ${ }^{1}$ Global TB Programme, World Health Organisation, Geneva, Switzerland. ${ }^{2}$ WHO Collaborating Centre for TB/HIV and TB Elimination, University of Brescia, Brescia, Italy. ${ }^{3}$ MRC Clinical Trials Unit and Centre for Infectious Disease Epidemiology, UCL, London, UK. ${ }^{4}$ Respiratory Infections Unit, Dept for Infectious Disease Epidemiology, Robert Koch Institute, Berlin, Germany. ${ }^{5}$ Dept of Infectious Diseases Galliera Hospital, Genoa, Italy. ${ }^{6}$ WHO Collaborating Centre for TB and Lung Diseases, Fondazione S. Maugeri, Care and Research Institute, Tradate, Italy.

Correspondence: Haileyesus Getahun, Global TB Programme, World Health Organization, 20 Avenue Appia $\mathrm{CH}-1211$ Geneva, Switzerland. E-mail getahunhawho.int

@ERSpublications

Intensification of \#LTBI treatment for child \#TB contacts and people living with \#HIV and other at risk populations http://ow.ly/ZfzAv

In this issue of the European Respiratory Journal, ERKENs et al. [1] present experience and results of long-term data (1993-2013) in the recording and reporting of latent tuberculosis (TB) infection (LTBI) management from the Netherlands. The authors report a high rate of initiation (77\%) among the 37729 eligible persons and a high rate of treatment completion (82\%). Valid and complete data were available on almost all (96\%) persons who started treatment, due to the use of a technically sound notification system supported by a legal framework and standardised indicators [1]. These findings are important additions to the renewed focus and call to address LTBI, which is considered as the reservoir for virtually all TB cases $[2,3]$. Other renewed efforts include the establishment of a World Health Organization (WHO) global task force to raise the profile of programmatic management of LTBI, including monitoring and evaluation of implemented activities, as well as promoting research. UNITAID has also identified LTBI as one of its areas of intervention.

The management of LTBI is a key component of the WHO End TB Strategy [4], which is critical to the advancement of TB control, particularly when TB elimination efforts are pursued [5]. Modelling showed that protection of $8 \%$ of people with LTBI each year, from developing active TB disease, could result in a 14-fold decrease of the global incidence of TB in 2050 compared to the incidence in 2013 [6].

\section{Two-pronged global approach}

WHO has issued global recommendations on the treatment of LTBI for people living with HIV [7] and for children aged $<5$ years who are close contacts of a pulmonary TB case [8]. Additionally, WHO recommends the management of LTBI among at risk populations in upper-middle and high-income countries with an estimated TB incidence rate of $<100$ per 100000 population [9]. In these countries, systematic LTBI testing and treatment is strongly recommended for a wider range of clinical risk groups: patients with silicosis, patients initiating anti-tumour necrosis factor (TNF) treatment, patients on dialysis, and patients preparing for organ and haematological transplantation. This two-prong approach and the tailored recommendations on diagnosis and treatment are presented in table 1.

Received: March 022016 | Accepted: March 022016

Conflict of interest: None declared.

The content of this work is copyright of the authors or their employers. Design and branding are copyright @ERS 2016. 
TABLE 1 Summary table of World Health Organization recommendations for latent tuberculosis (TB) infection (LTBI) programmatic management based on category of countries

\begin{tabular}{|c|c|c|c|}
\hline Country groups & $\begin{array}{l}\text { Indicated at risk populations } \\
\text { [strong recommendations] }\end{array}$ & Testing algorithm & Treatment options \\
\hline $\begin{array}{l}\text { High-income and upper-middle } \\
\text { income countries with an estimated } \\
\text { TB incidence rate }<100 \text { per } 100000 \\
\text { population (low TB burden) }\end{array}$ & $\begin{array}{l}\text { People living with HIV } \\
\text { Adults and children who are } \\
\text { household contacts of } \\
\text { pulmonary TB cases } \\
\text { Clinical indications: patients } \\
\text { with silicosis; patients initiating } \\
\text { anti-TNF treatment; patients on } \\
\text { dialysis; patients preparing for } \\
\text { organ or haematological } \\
\text { transplantation }\end{array}$ & $\begin{array}{l}\text { Exclude active TB using } \\
\text { TB investigations according } \\
\text { to national guidelines } \\
\text { A positive IGRA or TST result } \\
\text { is required to diagnose LTBI }\end{array}$ & $\begin{array}{l}6 \text { months daily isoniazid } \\
9 \text { months daily isoniazid } \\
3 \text { months weekly rifapent } \\
\text { plus isoniazid } \\
3-4 \text { months daily isoniazi } \\
\text { plus rifampicin } \\
\text { 3-4 months daily rifampi }\end{array}$ \\
\hline $\begin{array}{l}\text { Resource-limited and other high and } \\
\text { middle-income countries with an } \\
\text { estimated TB incidence rate }>100 \text { per } \\
100000 \text { population (high TB burden) }\end{array}$ & $\begin{array}{l}\text { People living with HIV } \\
\text { Children aged }<5 \text { years who } \\
\text { are household contacts of } \\
\text { a pulmonary TB case }\end{array}$ & $\begin{array}{l}\text { Exclude active TB using } \\
\text { TB investigations according } \\
\text { to national guidelines } \\
\text { An LTBI test is not required } \\
\text { prior to LTBI treatment, } \\
\text { but is encouraged for } \\
\text { people living with HIV } \\
\text { IGRA should not replace TST }\end{array}$ & 6 months daily isoniazid \\
\hline
\end{tabular}

TNF: tumour necrosis factor; IGRA: interferon- $\gamma$ release assay TST: tuberculin skin test.

\section{Barriers for programmatic management}

The programmatic management of LTBI should consider the underlying epidemiology of TB, the burden of LTBI among risk groups, the availability of national policies and surveillance as well as effective health service delivery system and resources [10]. However, there is a gap between the existence of a policy and the actual implementation of diagnosis and treatment of LTBI [11]. Furthermore, recommendations contained in national guidelines from low TB incidence countries differ in selection of risk groups and test methods as well as on treatment options [12], while in some high TB-burden countries restrictive national policies impede the implementation of treatment for LTBI [13]. Among people living with HIV in high TB-burden settings, significant barriers were present at different levels. In particular, at the programme level negative attitudes of managers and prominent opinion leaders, primarily due to concerns about the development of drug resistance [13], prevented implementation of LTBI treatment. While at the service delivery level, the lack of experience, knowledge and clarity on the benefits of preventive therapy and existing guidelines by health workers, hampered its utility [14]. Increased risk developing drug resistance, following treatment for latent TB, has not been established $[9,15]$.

The lack of a gold standard for LTBI diagnosis and imperfection of the acceptable tests to predict progression to active TB [16, 17], and long-winded treatment [12] are key elements in compromising programmatic management of LTBI. The tuberculin skin test (TST) is widely used and inexpensive but it has poor sensitivity in immunocompromised people [18], and is subject to cross reactivity with environmental non-TB mycobacteria [16]. Interferon- $\gamma$ release assays measure in vitro effector T-cell responses to two immunogenic Mycobacterium tuberculosis antigens $(6 \mathrm{kDa}$ early secretory antigenic target (ESTA-6) and $10 \mathrm{kDa}$ culture filtrate protein (CFP-10)], which are not found in bacille Calmette-Guerin and most non-tuberculous mycobacteria. However, these tests are expensive, requiring equipment and infrastructure [19]. A novel M. tuberculosis specific skin test, C-Tb, which contains ESAT-6 and CFP-10 antigens, has been developed and shown to have improved specificity [20] and sensitivity [21] than TST. However, the utility of this novel skin test has yet to be investigated for the diagnosis of latent TB infection.

Critical bottlenecks for programmatic management of LTBI include fragmented organisation of services for the identification of individuals, for testing and treating of eligible persons, as well as recording, reporting and follow-up of patients. For example, in most low TB burden countries, LTBI interventions among contacts are usually carried out by public health services, while a wide spectrum of clinical services handle LTBI management in clinical risk groups: infectious diseases services for people living with HIV; nephrology units for patients awaiting dialysis; rheumatology units for candidates with anti-TNF therapy; pneumology units for patients with silicosis; and a mixed variety of other specialties for candidates who will undergo transplantation. The lack of standardised indicators and case definitions applicable across these services 
impedes functional and integrated monitoring and evaluation systems for programmatic management of LTBI. A recent survey performed by the European Respiratory Society and WHO among 31 European countries shows that only six countries were able to report LTBI treatment completion rates, which ranged between 40 and $88 \%$ [22]. The role and feasibility of LTBI management among asylum seekers has also been raised in the face of recent influx of migrants to Europe, presenting another programmatic challenge [23].

\section{Solutions for programmatic management}

The implementation of the programmatic management of LTBI requires establishing national policies and legal frameworks, as well as close collaboration and harmonisation across the different services [9]. This was illustrated by the experience of ERKENs et al. [1] in the Netherlands that the adoption of the policy and legal framework coupled with the centralised web-based integration of the reporting systems with ongoing evaluation of its performance including clarifying the case definitions has been instrumental for the success of the programme [1]. Their ability to report the management of LTBI in clinical at risk groups, such as anti-TNF treatment candidates is particularly encouraging, and reflects the intensified collaboration between the municipality health and clinical services.

While there is no current mechanism to capture the provision of treatment for LTBI globally for at risk populations and child contacts, there has been a burgeoning experience in the global monitoring of the provision of isoniazid preventive treatment among people living with HIV (figure 1). This was possible by standardising the definition of the indicator to measure the number of adults and children living with HIV who are started on treatment for LTBI, expressed as a percentage of the total number of newly enrolled adults and children in HIV care during the reporting period [24], and mainstreaming this indicator to the existing HIV monitoring systems of WHO and UNAIDS (The Joint United Nations Programme on HIV/ AIDS) [25]. This was also enabled by the implementation of a four symptom-based simplified algorithm that has a negative predictive value $>98 \%$ to exclude active TB [7]. It is estimated that half of the people living with HIV who are newly enrolled into HIV care will fulfil this criteria and hence are eligible for TB preventive treatment [26].

It is, therefore, essential to develop standard indicators to accelerate and monitor the global implementation of programmatic management of LTBI, particularly for child contacts and other at risk populations. Such efforts should be based on a clear definition of numerators and denominators as well as the source of information and responsible services. Similarly, simplified algorithms should be used and developed to advance the programmatic management of LTBI among at-risk populations including child contacts. Estimating targets for eligible candidates for LTBI testing and treatment will be useful for programme management. These efforts will help to monitor the global progress in the implementation of programmatic management of LTBI.

Establishing effective systems for client education on adherence and clinical monitoring of adverse events, as well as supplying diagnostic tests and the drugs are other crucial steps. Programmes should design flexible interventions that are tailored to respond to the local context and needs of the population to ensure adherence to, and completion of, LTBI treatment. Furthermore, establishing continuous surveillance and evaluating the impact of the programmatic management is important as well.

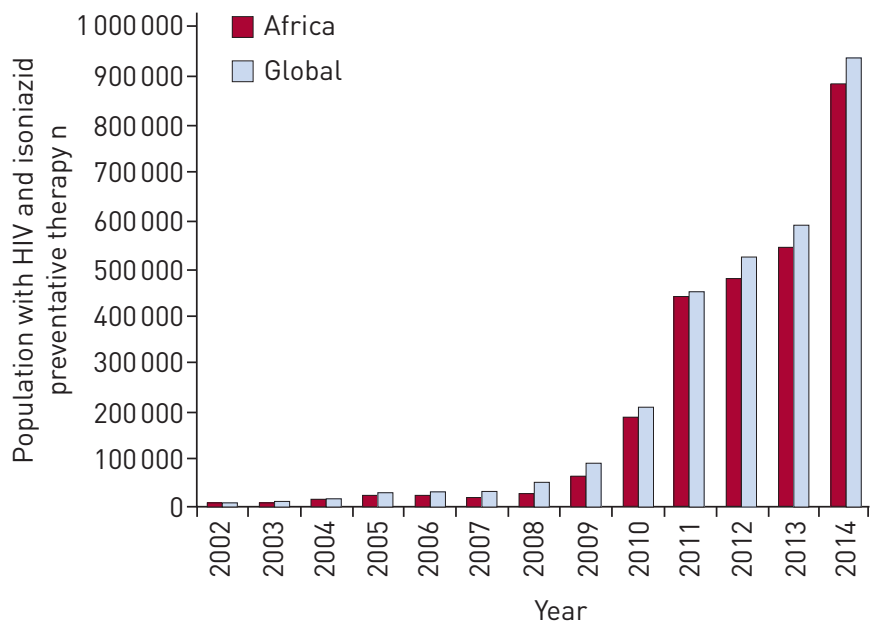

FIGURE 1 The number of people living with HIV and who have been initiated on isoniazid preventive therapy both globally and in Africa alone, between 2002-2014. 
Operational research efforts to enable the effective delivery of the intervention based on context and disease epidemiology need to be an integral part of programmatic management of LTBI.

\section{References}

1 Erkens CGM, Slump E, Verhagen $\mathrm{M}$ et al. Monitoring latent tuberculosis infection diagnosis and management in the Netherlands. ERJ 2016; 47: 1492-1501.

2 Getahun H, Chaisson RE, Raviglione M. Latent Mycobacterium tuberculosis infection. N Engl J Med 2015; 373: $1179-1180$.

3 Rangaka MX, Cavalcante SC, Marais BJ, et al. Controlling the seedbeds of tuberculosis: diagnosis and treatment of tuberculosis infection. Lancet 2015; 386: 2344-2353.

4 Uplekar M, Weil D, Lonnroth K, et al. WHO's new end TB strategy. Lancet 2015; 385: 1799-1801.

5 Lönnroth K, Migliori GB, Abubakar I, et al. Towards tuberculosis elimination: an action framework for low-incidence countries. Eur Respir J 2015; 45: 928-952.

6 Dye C, Glaziou P, Floyd K, et al. Prospects for tuberculosis elimination. Annu Rev Public Health 2013; 34: 271-286.

7 World Health Organization. Guidelines for Intensified Tuberculosis Case-Finding and Isoniazid Preventive Therapy for People Living with HIV in Resource-Constrained Settings. Geneva, World Health Organization, 2011. Available from: http://apps.who.int/iris/bitstream/10665/44472/1/9789241500708_eng.pdf

8 World Health Organization. Recommendations for Investigating Contacts of Persons with Infectious Tuberculosis in Low-and Middle-Income Countries. Geneva, World Health Organization, 2012. Available from: www.who.int/ tb/publications/2012/contact_investigation2012/en/

9 Getahun H, Matteelli A, Abubakar I, et al. Management of latent Mycobacterium tuberculosis infection: WHO guidelines for low tuberculosis burden countries. Eur Respir J 2015; 46: 1563-1576.

10 World Health Organization. Guidelines on the Management of Latent Tuberculosis Infection. Geneva, World Health Organization, 2015. Available from: www.who.int/tb/publications/ltbi_document_page/en/

11 World Health Organization. Global Tuberculosis Report 2015. 20th Edn. Geneva, World Health Organisation, 2015. Available from: www.who.int/tb/publications/global_report/en/

12 Getahun H, Matteelli A, Chaisson R, et al. Latent Mycobacterium tuberculosis infection. N Engl J Med 2015: 372: 2127-2135.

13 Getahun H, Granich R, Sculier D, et al. Implementation of isoniazid preventive therapy for people living with HIV worldwide: barriers and solutions. AIDS 2010; 24: Suppl. 5, S57-S65.

14 Lester R, Hamilton R, Charalambous S, et al. Barriers to implementation of isoniazid preventive therapy in HIV clinics: a qualitative study. AIDS 2010; 24: Suppl. 5, S45-S48.

15 Balcells ME, Thomas SL, Godfrey-Faussett P, et al. Isoniazid preventive therapy and risk for resistant tuberculosis. Emerg Infect Dis 2006; 12: 744-751.

16 Pai M, Denkinger CM, Kik SV, et al. Gamma interferon release assays for detection of Mycobacterium tuberculosis infection. Clin Microbiol Rev 2014; 27: 3-20.

17 Diel R, Goletti D, Ferrara G, et al. Interferon- $\gamma$ release assays for the diagnosis of latent Mycobacterium tuberculosis infection: a systematic review and meta-analysis. Eur Respir J 2011; 37: 88-99.

18 O'Garra A, Redford PS, McNab FW, et al. The immune response in tuberculosis. Annu Rev Immunol 2013; 31: 475-527.

19 Dorman SE, Belknap R, Graviss EA, et al. Interferon- $\gamma$ release assays and tuberculin skin testing for diagnosis of latent tuberculosis infection in healthcare workers in the United States. Am J Respir Crit Care Med 2014; 189: 77-87.

20 Aggerbeck H, Giemza R, Joshi P, et al. Randomised clinical trial investigating the specificity of a novel skin test (C-Tb) for diagnosis of M. tuberculosis infection. PLoS One 2013; 8: e64215.

21 Hoff ST, Peter JG, Theron G, et al. Sensitivity of C-Tb: a novel RD-1-specific skin test for the diagnosis of tuberculosis infection. Eur Respir J 2016; 47: 919-928.

22 D'Ambrosio L, Dara M, Tadolini M, et al. Tuberculosis elimination: theory and practice in Europe. Eur Respir J 2014; 43: 1410-1420.

23 de Vries G, van Rest J, Meijer W, et al. Low yield of screening asylum seekers from countries with a tuberculosis incidence of $<50$ per 100000 population. Eur Respir J 2016 [in press; DOI: 10.1183/13993003.00099-2016].

24 World Health Organization. A Guide to Monitoring and Evaluation for Collaborative TB/HIV Activities. Geneva, World Health Organization, 2015. Available from: www.who.int/tb/publications/m_and_e_document_page/en/

25 World Health Organization. Three Interlinked Patient Monitoring Systems for HIV and TB/HIV: Standardized Minimum Data Set and Illustrative Tools. Geneva, World Health Organization, 2013. Available from: www.who. int/hiv/pub/me/patient_monitoring_systems/en/

26 Getahun H, Kittikraisak W, Heilig CM, et al. Development of a standardized screening rule for tuberculosis in people living with HIV in resource-constrained settings: individual participant data meta-analysis of observational studies. PLoS Med 2011; 8: e1000391. 